\section{REVISTA}

MEXICANA DE

ECONOMÍA Y

FINANZAS

REMEF

(TIIE MEXICAN JOURNAL OF

ECONOMICS AND FINANCE
Revista Mexicana de Economía y Finanzas, Nueva Época

Volumen 17 Número 2, Abril - Junio 2022, pp. 1-21, e606

DOI: https://doi.org/10.21919/remef.v17i2.606

(Recibido: 26/febrero/2021, aceptado: 2/julio/2021, publicado: $20 /$ diciembre/2021)

\title{
La hipótesis de convergencia en México: un enfoque de $\sigma$-convergencia débil
}

\author{
Domingo Rodríguez Benavides ${ }^{1}$ - Universidad Autónoma Metropolitana, México \\ Miguel Ángel Mendoza González² - Universidad Nacional Autónoma de México, México \\ José Antonio Climent Hernández - Universidad Autónoma Metropolitana, México
}

Se analiza la hipótesis de convergencia en México con la prueba relativamente novedosa de $\sigma$-convergencia débil, propuesta por Kong, Phillips y Sul (2019), en el periodo 1940-2019 y en dos subperiodos con la totalidad de las entidades federativas y con grupos formados a partir de su niveles de ingreso, con el fin de determinar si el proceso de liberalización comercial y la entrada en vigor del Tratado de Libre Comercio de América del Norte (TLCAN) promovieron la convergencia regional. Se encuentra que tanto para el primer periodo, como para todo el periodo hay evidencia de $\sigma$-convergencia débil, por el contrario revelan evidencia de divergencia para el segundo periodo. La aplicación de esta prueba en los grupos formados a partir de sus niveles de ingreso contribuye a identificar si algunos de estos grupos puede ser los causantes de la ausencia de convergencia de todas las entidades federativas. No obstante, una limitación de la misma es que puede no conciliar resultados obtenidos para todo el periodo en relación con los de los subperiodos.

Clasificación JEL: C33, C80, D63, R11.

Palabras clave: $\sigma$-convergencia, PIB per capita, Desigualdad Regional, México.

\section{The Convergence Hypothesis in Mexico: A Weak $\sigma$-Convergence Approach}

We analyze the convergence hypothesis in Mexico with the relatively novel test of -weak convergence, proposed by Kong, Phillips and Sul (2019), in the period 1940-2019 and in two sub-periods with the totality of the federal entities and with groups formed from their income levels, in order to determine whether the process of trade liberalization and the entry into force of the North American Free Trade Agreement (NAFTA) promoted regional convergence. It is found that for both the first period and the whole period there is evidence of weak convergence, on the contrary, evidence of divergence is revealed for the second period. The application of this test to groups formed on the basis of their income levels helps to identify whether some of these groups may be responsible for the lack of convergence of all the states. However, a limitation of this test is that it may not reconcile results obtained for the whole period with those of the sub-periods.

JEL Classification: C33, C80, D63, R11.

Keywords: $\sigma$-convergence, GDP per capita, Regional Inequality, Mexico.

${ }^{1}$ Autor de correspondencia. Departamento de Sistemas, UAM-Azcapotzalco, e-mail: dorobe@azc.uam.mx

*Sin fuente de financiamiento para el desarrollo de la investigación

${ }^{2}$ Agradece el financiamiento del proyecto PAPIIT-IN308721-UNAM “Políticas públicas de reactivación y reestructuración económica urbana en México ante impactos económicos y sociales de la COVID-19 en México". 


\section{Introducción}

La hipótesis de la convergencia económica regional continúa siendo el centro de una gran cantidad de trabajos de investigación sobre el crecimiento económico que se han debatido en la literatura durante más de medio siglo. En deuda con el modelo de crecimiento neoclásico por sus raíces teóricas, el debate sobre el concepto de convergencia se caracteriza por diversos hallazgos empíricos que se basan en una amplia gama de metodologías econométricas. Los estudios iniciales sobre el tema fueron independientes del marco del modelo de crecimiento neoclásico. Sin embargo, Barro y Sala-i-Martin (1990) derivaron la ecuación de convergencia del modelo de crecimiento neoclásico y a dicha hipótesis se le denominó $\beta$-convergencia que, debido al supuesto de estados estacionarios constantes entre las regiones, se le conoce como convergencia $\beta$-absoluta o incondicional. Este tipo de convergencia implica una relación negativa entre el ingreso inicial per cápita y el crecimiento posterior, asumiendo estados estacionarios constantes en todas las unidades transversales. Friedman (1992) fue uno de los primeros críticos del análisis de regresión de la $\beta$-convergencia incondicional. Se opuso a las estimaciones transversales de la ecuación de convergencia porque se basan en un promedio para todo el período de tiempo. La conclusión de la convergencia de ingresos basada en este último se denominó "falacia de la regresión", en lugar de ser un indicativo de que los pobres se vuelven ricos. Como alternativa a la $\beta$-convergencia, Friedman (1992) propuso el estudio temporal de la dispersión transversal del ingreso que es más plausible. Sin embargo, incluso antes de la recomendación de Friedman, la tendencia de la dispersión del ingreso entre países se analizó en la convergencia empírica junto con la $\beta$-convergencia. Además, la ecuación para $\sigma$-convergencia, como se le denomina, se derivó de la de la $\beta$-convergencia, esta última se presenta como una condición necesaria pero no suficiente para la primera, Barro y Sala-i-Martin (1990), (1992), (2004).

En la investigación empírica reciente sobre el tema, México representa un interesante caso para los estudiosos de la convergencia económica en virtud de que desde mediados del siglo pasado ha atravesado por varias etapas de desarrollo, un proceso de sustitución de importaciones (19601985) seguido de una era de liberalización comercial (de 1985 al presente) además de una relocalización de su planta productiva en la que el país desplazó la industria fuera del área central del país (Estado de México y Ciudad de México) a la región fronteriza del norte (Baja California, Sonora, Chihuahua, Coahuila, Nuevo León y Tamaulipas), Brock y German-Soto (2013), Becerril et al. (2010), Jordaan y Rodríguez-Oreggia (2012), Rodríguez-Oreggia (2005, 2007). Además, la literatura económica sobre convergencia ha identificado diversos mecanismos a través de los cuales las reformas comerciales han tenido efectos diferenciados en las regiones de México, Chiquiar (2005).

Por otro lado, Mendoza, German, Monfort y Ordoñez (2020) argumentan que el estudio de la convergencia regional en México es relevante por diversas razones entre las que destacan $i$ ) posee una de las mayores tasas de desigualdad entre los países de la OCDE; ii) los cambios políticos, económicos, demográficos e institucionales que ha experimentado a lo largo de su historia; iii) las tendencias de su desigualdad han sido sustancialmente diferentes de las observadas en otros países en desarrollo; y iv) el impacto heterogéneo en las regiones de la internacionalización de la economía.

En este trabajo se emplea una prueba relativamente novedosa para probar convergencia conocida como $\sigma$-convergencia débil propuesta por Kong, Phillips y Sul (2019), la cual permite probar distintas formas de variabilidad decreciente en los datos a través de un modelo de regresión. 
La metodología se basa en un estadístico de prueba $t$ simple que permite identificar convergencia a pesar de que la regresión este incorrectamente especificada bajo una variabilidad decreciente.

El resto del trabajo se organiza de la siguiente manera. En la sección II se brinda una breve revisión de los trabajos que se han realizado con el fin de probar la hipótesis de convergencia en México. La sección III describe la metodología econométrica empleada. La sección IV muestra los resultados, y por último, la sección final concluye.

\section{Revisión de la literatura empírica}

Con el fin de probar distintas hipótesis de convergencia regional en México se ha hecho un notable esfuerzo por construir bases de datos históricas que cubran mayores periodos de tiempo, ejemplo de ello son los trabajos de Esquivel (1999), German-Soto (2005), Mendoza (2014) y Aguilar-Retureta (2010 y 2016) entre otros, ya que las bases de datos oficiales con las que se cuenta cubren periodos de tiempo relativamente cortos o bien se dispone sólo para algunos años. En este trabajo empleamos la base de datos del PIB por habitante de 1940 a 2019 cuya metodología de generación se remite a German-Soto (2005).

Diversos trabajos han empleado el análisis de convergencia beta tradicional, la mayoría de ellos encuentran una ralentización de la convergencia y divergencia, Juan-Ramon y Rivera Batiz (1996), Esquivel (1999), Messmacher (2000), Cermeño (2001), Esquivel y Messmacher (2002) y Chiquiar, (2005).

Entre los trabajos que han analizado convergencia en México con los enfoques de ( $\beta$ convergencia y $\sigma$-convergencia se encuentran los de Mallick y Carayannis (1994), Juan Ramón y Rivera Bátiz (1996), Esquivel (1999), Messmacher (2000), Rodríguez Oreggia (2001), Cabrera (2002), García Verdú (2002) y Rodríguez-Pose y Sánchez-Reaza (2002a) quienes estudian la convergencia absoluta entre los estados mexicanos. Todos tienden a concluir que hay evidencia de convergencia en México a nivel de entidades federativas hasta mediados de los ochenta, pero que este patrón se rompió posteriormente. No obstante, la mayoría de estos estudios comprenden periodos de análisis de crecimiento regional hasta 1996, por lo que no analizan la experiencia posterior al TLCAN.

Messmacher (2000) y Rodríguez-Pose y Sánchez-Reaza (2002a), analizan la convergencia durante el período posterior al TLCAN con los patrones observados anteriormente. Los resultados de Rodríguez-Pose y Sánchez-Reaza (2002a) sugieren que el TLCAN no restableció la convergencia. Los resultados de Messmacher (2000), sin embargo, contradicen los hallazgos de estos autores en virtud de que no obtiene estimaciones estadísticamente significativas para el parámetro de convergencia $\beta$ en ninguno de los periodos muestrales. Esquivel y Messmacher (2002) muestran que el comportamiento de la productividad laboral fue una fuente importante de convergencia en el producto per cápita entre 1960 y 1990. Por el contrario, la productividad laboral fue el principal factor detrás del patrón de divergencia regional en el producto per cápita observada en la década de los noventa.

Chiquiar (2005) encuentra que el patrón divergente observado después de 1985 no se revirtió con el TLCAN y que los estados que se beneficiaron con las reformas fueron aquellos que 
inicialmente estaban mejor dotados y que fueron más capaces de atraer mayores niveles de capital físico y humano, así como una mejor infraestructura.

Plata, Calderón y Salazar (2013) encuentran evidencia de convergencia a nivel de entidades federativas en México, pero a una velocidad inferior, de 1.1\% en promedio, a la reportada en otros estudios a nivel internacional que es de aproximadamente del 2\%. Kido-Cruz y Kido-Cruz (2015) rechazan las hipótesis de convergencia absoluta y condicional en cuatro estados de la República Mexicana: Chiapas, Guerrero. Michoacán y Oaxaca en el periodo de 1990 a 2010. German, Rodríguez y Gallegos (2020) investigan el proceso de convergencia regional en México a nivel de estados a través del enfoque de $\beta$-convergencia en el periodo 1940-2015, sus resultados muestran evidencia de $\beta$-convergencia regional y que la velocidad en la que se registra es mayor para los estados más expuestos a la globalización y menor para los menos expuestos a dicho proceso.

Fonseca, Llamosas y Rangel (2018) analizan la hipótesis de convergencia durante el periodo 1994-2015, periodo en el cual consideran el impacto del tratado de libre comercio de América del Norte entre otros factores y encuentran presencia de divergencia absoluta, consistente con un proceso de sigma divergencia particularmente en el periodo después de la ruptura que representó la crisis del 2008, además de ubicar un proceso de convergencia condicional en los subperiodos analizados por los autores.

Brock (2019) examina empíricamente la convergencia a nivel de municipios dentro de la región sur de Oaxaca de México. Encuentra que los municipios están convergiendo más rápidamente que la "ley del hierro" del $2 \%$ con hasta una velocidad de $\beta$ convergencia de $8 \%$.

Otra serie de trabajos se han enfocado en mostrar evidencia de convergencia estocástica en México, la cual se prueba básicamente a través de pruebas de raíces unitarias ya sea de manera univariada o en panel. Por ejemplo, Carrion-i-Silvestre y Germán-Soto (2007 y 2009) a través de pruebas de raíces unitarias analizan el proceso de convergencia estocástica en México, sus resultados muestran que dicho proceso no se puede entender si no se considera el cambio estructural registrado en la década de los ochentas.

Gómez y Ventosa-Santaulària (2009) analizan la convergencia entre regiones de la República Mexicana en relación a la capital del país, encuentran evidencia de que la diferencia en ingreso per cápita de cada estado tiende a disminuir a través del tiempo. De igual forma encuentran evidencia de que las reformas comerciales de mediados de los años ochenta afectaron negativamente el proceso de convergencia para algunas regiones, principalmente las más pobres, tampoco encuentran evidencia que les permita concluir que la apertura comercial promovió convergencia entre las regiones fronterizas de México y Estados Unidos. En el mismo sentido, Sánchez-Reaza y RodríguezPose (2002), Rodríguez-Pose y Sánchez-Reaza (2005) y Villarreal y Tykhonenko (2007) encuentran que los estados mexicanos que realizan más comercio con Estados Unidos crecieron más rápido que los demás, pero que no hubo un cambio significativo en este patrón después de la firma del TLCAN.

Díaz, Sánchez y Mendoza (2009) proveen evidencia empírica respecto a la convergencia del ingreso per cápita de los estados de la República Mexicana con respecto a la economía líder a través de pruebas de raíces unitarias y de cointegración en panel. Los resultados de las pruebas de raíces unitarias no respaldan la hipótesis de convergencia absoluta hacia la economía líder mientras que las pruebas de cointegración muestran evidencia que favorece la hipótesis de convergencia condicional. 
Gómez-Zaldivar y Ventosa-Santaulària (2010) a través de distintas pruebas de raíces unitarias con rupturas analizan la hipótesis de la convergencia, sus resultados muestran evidencia en favor de la divergencia.

Gómez-Zaldivar y Ventosa-Santaulària (2012) examinan la conducta de las disparidades a nivel de regiones en México en relación a la región más rica, la Ciudad de México, durante el periodo 1940-2009, sus resultados muestran que mientras algunas regiones han mostrado un proceso de Catching-up la región fronteriza de México se ha rezagado con respecto a su contraparte estadounidense.

Rodríguez, Mendoza y Venegas (2016a) evalúan la hipótesis de convergencia regional en México a través de un modelo de crecimiento no lineal, específicamente a través de un modelo panelTAR. Sus resultados muestran evidencia de convergencia parcial y absoluta para el grupo de las 11 entidades consideradas como las "más ricas" en determinados periodos.

Rodríguez, Trejo y Mendoza (2016c) analizan la convergencia en el PIB per cápita de las entidades de la República Mexicana en el periodo 1940-2010 a través del enfoque de pares propuesto por Pesaran (2007). Sus resultados muestran evidencia que va en contra de la hipótesis de convergencia para todo el periodo muestral, que va de 1940 a 2010, y también para el primer periodo de análisis que va de 1940 a 1985 . No obstante, encuentran evidencia mixta de dicha hipótesis en el segundo periodo en el que dividen la muestra, el cual corresponde de 1986 a 2010.

Mendoza, Ventosa-Santaulària y German (2019) a través de una batería de pruebas de raíces unitarias brindan evidencia sobre el proceso de convergencia de la desigualdad interregional en México, sus resultados muestran que la mayoría de las regiones divergen o se están poniendo al día (catching-up).

Uno de los inconvenientes de las pruebas de raíces unitarias para probar la hipótesis de convergencia es que proporcionan resultados engañosos si los datos contienen dinámicas de transición (o combinan tanto estados estacionarios como dinámicas de transición), Mendoza, German, Monford y Ordoñez (2020). Adicionalmente, si está presente más de un equilibrio, este tipo de pruebas fracasan para detectar convergencia, Apergis, Christou y Miller (2012).

Rodríguez, López y Mendoza (2016c) investigan la hipótesis de convergencia relativa en el PIB per cápita de las entidades federativas de la República Mexicana en el periodo 1970-2012 a través de la metodología empleada por Phillips y Sul (2007), encuentran evidencia de convergencia relativa en seis grupos o clubes de convergencia. Mendoza, German, Monford y Ordoñez (2020) emplean la misma metodología con datos de ingreso per cápita de las entidades federativas en el periodo 19402015, sus resultados tienden a corroborar la existencia de clubes de convergencia en ambos indicadores.

Trejo (2020) mide las disparidades regionales de México y el desempeño económico nacional en el periodo 1940-2013 con el fin de verificar si los desequilibrios regionales se expanden o se contraen con las fases del ciclo económico agregado y también si dichas disparidades aumentan o se reducen de acuerdo a la etapa de desarrollo del país. Sus resultados muestran que no hay evidencia que soporte la evolución prociclica de las desigualdades regionales.

Recientemente, se han empleado metodologías novedosas que incorporan efectos espaciales en el proceso de convergencia. En este contexto, Díaz, Fernández, Garduño y Rubiera (2019) a través de un modelo de $\beta$ convergencia condicional espacial prueban la hipótesis de convergencia regional con datos a nivel de entidad federativa y de municipio en el periodo de 1980 a 2008. Sus resultados 
muestran que para el periodo previo al TLCAN hay un proceso de convergencia alentado por las entidades de la región fronteriza mientras que para el segundo periodo no encontraron evidencia de convergencia.

Valdez (2019) estima el impacto de las externalidades espaciales del capital en la producción. Sus resultados muestran que la difusión espacial del crecimiento económico está relacionada directamente con la distancia y encuentran evidencia a favor de la hipótesis de convergencia. Adicionalmente muestra que la distancia entre los estados estacionarios es insuficiente para explicar las diferencias entre las tasas de crecimiento, sino que también es importante considerar la distancia geográfica. Rodríguez y Cabrera (2019) investigan si la contigüidad geográfica es importante en el proceso de convergencia (absoluta y condicional) a nivel de municipios en México en el periodo 1999-2014. Sus resultados muestran que los municipios pobres crecieron más que los ricos, principalmente en el último periodo de la muestra y que la dimensión espacial es un elemento cuya importancia se reduce a través del tiempo. De igual forma, sus resultados les permiten prever un crecimiento más autónomo de las economías en años recientes. Rodríguez y Cabrera (2020) investigan el patrón espacial de la convergencia en los municipios de México en el periodo que va de 1999 a 2014 a través de la regresión geográficamente ponderada. Sus resultados muestran evidencia de convergencia no estacionaria y proveen evidencia de un proceso heterogéneo y geográficamente diferenciado.

\section{Los datos}

Los datos del PIB per cápita de 1940 a 2015 se contruyeron a partir de la base datos tanto del PIB como de la población por entidad federativa de Germán-Soto (2005) y Germán-Soto (2014), respectivamente, y se actualizaron en el periodo 2015-2019 aplicando las tasas de crecimiento de los indicadores trimestral de la actividad económica estatal (ITAEE) ajustada al PIB de cada entidad federativa para obtener el crecimiento nacional observado de acuerdo con el INEGI. En tanto, para actualizar la serie de población por entidad federativa de 1940 a 2010 de Germán-Soto (2015) se utilizaron tasas de interpolación anual de acuerdo con los datos del censo de población 2020. Con la finalidad de aportar evidencia adicional de manera desagregada, además de efectuar la prueba con la totalidad de las entidades federativas y cuando se excluyen las entidades petroleras en todo el periodo como en subperiodos, optamos por clasificar a las entidades federativas en terciles. Los resultados de la clasificación por terciles para las entidades federativas, excluyendo las entidades federativas petroleras, se muestran en el cuadro 1.

En el cuadro 1 también se muestra el PIB per cápita de cada entidad al principio y al final de cada periodo de análisis, el grupo de ingreso al que pertenece de acuerdo con los años 1986 y 1995, así como las tasas de crecimiento promedio por periodo de análisis. Alrededor de dos terceras partes de las entidades federativas se mantuvieron en el tercil en el que fueron ubicadas en el año de 1986, entre las entidades que bajaron de posición se encuentran Colima, Jalisco, Nayarit y Veracruz mientras que las que mejoraron su posición son Aguascalientes, Guanajuato, Querétaro y San Luis Potosí. 
Cuadro 1. PIB por habitante por entidades federativas

Miles de pesos de 1993

\begin{tabular}{|c|c|c|c|c|c|c|c|c|c|c|}
\hline \multirow{2}{*}{ Entidades federativas } & \multicolumn{4}{|c|}{$\begin{array}{l}\text { PIB por habitante } \\
\text { promedio }\end{array}$} & \multicolumn{2}{|c|}{ Grupos de ingreso } & \multicolumn{4}{|c|}{$\begin{array}{l}\text { Tasa de crecimiento } \\
\text { promedio anual }\end{array}$} \\
\hline & 1940 & 1986 & 1995 & 2019 & 1986 & 1995 & $\begin{array}{l}1940- \\
1985\end{array}$ & $\begin{array}{l}1940- \\
1994\end{array}$ & $\begin{array}{l}1986- \\
2019\end{array}$ & $\begin{array}{l}1995 \\
2019\end{array}$ \\
\hline Aguascalientes & 4.1 & 11.0 & 13.7 & 25.8 & medio & alto & 2.2 & 2.4 & 2.6 & 2.7 \\
\hline Baja California & 16.4 & 18.4 & 15.5 & 19.9 & alto & alto & 0.1 & 0.1 & 0.2 & 1.1 \\
\hline Baja California Sur & 3.8 & 30.2 & 16.8 & 20.2 & alto & alto & 3.2 & 2.9 & -1.2 & 0.8 \\
\hline \multicolumn{11}{|l|}{ Campeche } \\
\hline Coahuila & 6.1 & 13.8 & 16.0 & 24.7 & alto & alto & 2.1 & 1.9 & 1.8 & 1.8 \\
\hline Colima & 5.1 & 13.5 & 13.2 & 18.3 & alto & medio & 2.3 & 1.9 & 0.9 & 1.4 \\
\hline Chiapas & 1.5 & 8.3 & 6.0 & 5.1 & bajo & bajo & 4.0 & 2.6 & -1.4 & -0.6 \\
\hline Chihuahua & 4.6 & 14.1 & 16.0 & 28.2 & alto & alto & 2.4 & 2.5 & 2.1 & 2.4 \\
\hline Ciudad de México & 14.7 & 24.0 & 30.7 & 48.1 & alto & alto & 1.2 & 1.6 & 2.1 & 1.9 \\
\hline Durango & 5.5 & 10.0 & 10.5 & 15.0 & medio & medio & 1.7 & 1.3 & 1.2 & 1.5 \\
\hline Guanajuato & 1.9 & 7.9 & 8.9 & 15.2 & bajo & medio & 3.6 & 3.0 & 2.0 & 2.3 \\
\hline Guerrero & 1.3 & 7.2 & 7.3 & 8.8 & bajo & bajo & 4.0 & 3.4 & 0.6 & 0.8 \\
\hline Hidalgo & 2.0 & 7.7 & 7.5 & 9.6 & bajo & bajo & 3.4 & 2.8 & 0.7 & 1.0 \\
\hline Jalisco & 2.5 & 13.0 & 12.1 & 17.5 & alto & medio & 3.9 & 3.2 & 0.9 & 1.6 \\
\hline México & 1.9 & 11.8 & 9.7 & 14.1 & medio & medio & 4.4 & 3.4 & 0.5 & 1.6 \\
\hline Michoacán & 1.4 & 7.0 & 7.3 & 10.4 & bajo & bajo & 3.7 & 3.1 & 1.2 & 1.5 \\
\hline Morelos & 3.3 & 10.1 & 11.0 & 15.8 & medio & medio & 2.8 & 2.6 & 1.4 & 1.5 \\
\hline Nayarit & 2.6 & 9.5 & 7.8 & 10.3 & medio & bajo & 3.1 & 2.3 & 0.3 & 1.2 \\
\hline Nuevo León & 6.6 & 18.9 & 20.6 & 32.7 & alto & alto & 2.6 & 2.3 & 1.7 & 2.0 \\
\hline Oaxaca & 0.8 & 6.8 & 5.9 & 6.7 & bajo & bajo & 4.8 & 3.9 & 0.0 & 0.5 \\
\hline Puebla & 1.6 & 8.1 & 7.8 & 12.2 & bajo & bajo & 3.9 & 3.2 & 1.3 & 1.9 \\
\hline Querétaro & 4.4 & 12.7 & 13.6 & 22.6 & medio & alto & 2.6 & 2.3 & 1.8 & 2.1 \\
\hline Quintana Roo & 7.9 & 13.2 & 21.0 & 23.6 & alto & alto & 1.5 & 2.1 & 1.8 & 0.5 \\
\hline San Luis Potosí & 2.2 & 8.0 & 8.8 & 16.3 & bajo & medio & 3.2 & 2.9 & 2.2 & 2.6 \\
\hline Sinaloa & 3.7 & 10.7 & 10.8 & 15.5 & medio & medio & 2.5 & 2.1 & 1.1 & 1.5 \\
\hline Sonora & 5.2 & 18.2 & 15.2 & 22.7 & alto & alto & 2.5 & 2.1 & 0.7 & 1.7 \\
\hline \multicolumn{11}{|l|}{ Tabasco } \\
\hline Tamaulipas & 6.0 & 12.7 & 12.9 & 18.4 & medio & medio & 1.8 & 1.6 & 1.1 & 1.5 \\
\hline Tlaxcala & 1.7 & 9.0 & 6.7 & 8.7 & bajo & bajo & 4.0 & 2.7 & -0.1 & 1.1 \\
\hline Veracruz & 3.5 & 9.5 & 8.1 & 10.0 & medio & bajo & 2.3 & 1.6 & 0.2 & 0.9 \\
\hline Yucatán & 4.4 & 9.5 & 9.6 & 14.8 & medio & medio & 1.7 & 1.6 & 1.4 & 1.8 \\
\hline Zacatecas & 1.7 & 6.3 & 7.4 & 11.2 & bajo & bajo & 3.4 & 2.8 & 1.8 & 1.7 \\
\hline
\end{tabular}

Nota: Los grupos de ingreso se construyeron con los terciles del PIB por habitante de 1986 y 1995; y, no se consideraron las entidades petroleras de Campeche y Tabasco

La figura 1 muestra las entidades federativas clasificadas de acuerdo a los grupos de ingreso alto, medio y bajo de acuerdo a su PIB per cápita correspondientes a los años de 1985 y 1986 . En ambas distribuciones espaciales se puede ver cierto agrupamiento en el que las entidades identificadas como de ingreso alto tienden a ubicarse en el norte del país y algunas entidades del 
centro del país que cambiaron de posición de 1986 a 1995 y la Ciudad de México y Quintana Roo. En tanto que las entidades clasificadas como de ingreso medio tienden a ubicarse en la zona central del país, con excepción de Tamaulipas y Yucatán. Y las entidades consideradas como de ingreso bajo se concentran en el sur y sureste de la Republica Mexicana además de Nayarit y Zacatecas. Está distribución espacial que presentan las entidades federativas de México destacan la importancia de considerar el factor espacio en los estudios empíricos, no obstante, en este trabajo no tomamos en cuenta este aspecto.

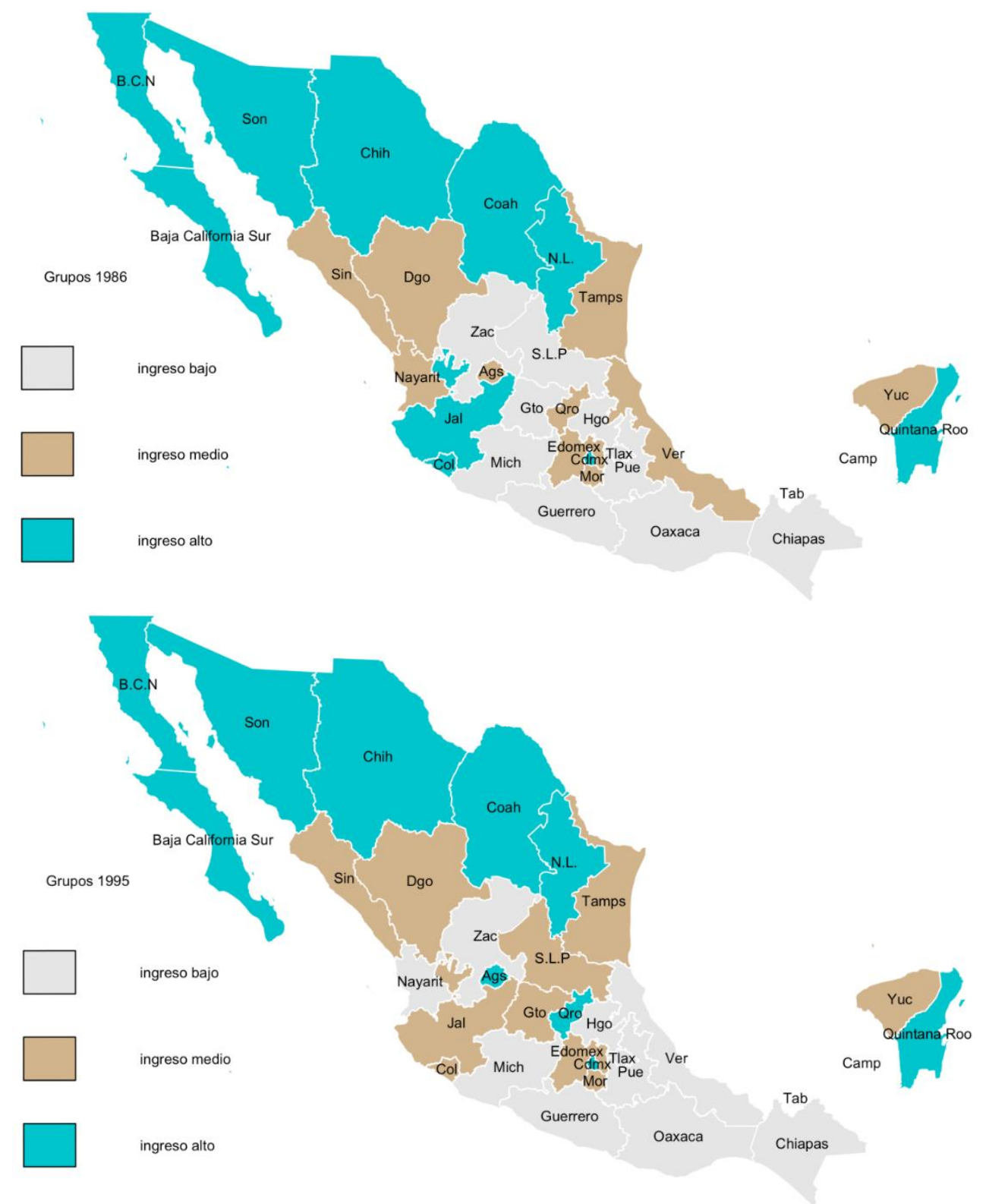

Figura 1. Grupos de entidades por ingreso, clasificaciones 1986 y 1995

Miles de pesos de 1993

Nota: Los grupos de ingreso se construyeron con los terciles del PIB por habitante de 1986 y 1995; y, no se consideraron las entidades petroleras de Campeche y Tabasco 
El cuadro 2 muestra un análisis descriptivo por grupos de ingreso, clasificados de acuerdo con los años 1986 y 1995, en el cual se puede apreciar de que independientemente del año en el que son clasificadas las entidades federativas las tasas de crecimiento promedio anual son mayores para el periodo que comprende de 1940 a 1985 principalmente para las entidades clasificadas como de ingreso bajo, lo cual se cumple también cuando el primer periodo de análisis se extiende hasta 1994, de igual forma es preciso destacar que estas tasas de crecimiento promedio anual registradas por los entidades clasificadas como de ingreso bajo son mayores que las que experimentaron los grupos de ingreso medio y alto. Este comportamiento de las tasas de crecimiento promedio anual de las entidades de ingreso bajo es consistente con la hipótesis de convergencia en el sentido de que muestra evidencia de que las entidades con menores ingresos crecieron a una mayor tasa a la que crecieron las entidades catalogadas como de ingreso alto.

Otro punto a destacar del análisis descriptivo que se presenta en el cuadro 2, es el hecho de que en el segundo periodo, independientemente de donde se ubique el año de ruptura o quiebre ya sea en 1986 o en 1995, los grupos de ingreso medio y alto crecieron a una tasa mayor que las entidades consideradas como de ingreso bajo, lo cual también refuerza la idea de la ausencia de convergencia resportada para este segundo periodo en el que comúnmente se divide la muestra de análisis.

Cuadro 2. Grupos de entidades por ingreso, clasificaciones 1986 y 1995

Miles de pesos de 1993

\begin{tabular}{|l|c|c|c|c|c|c|c|c|}
\hline \multirow{2}{*}{$\begin{array}{l}\text { Grupos de entidades } \\
\end{array}$} & \multicolumn{4}{|c|}{$\begin{array}{c}\text { PIB por habitante } \\
\text { promedio }\end{array}$} & \multicolumn{5}{c|}{$\begin{array}{c}\text { Tasa de crecimiento } \\
\text { promedio anual }\end{array}$} \\
\hline Clasificación de 1986 & 1940 & 1986 & 1995 & 2019 & $1940-1985$ & $1940-1994$ & $1986-2019$ & $1995-2019$ \\
\hline Ingreso bajo & 1.7 & 7.8 & 7.4 & 10.0 & 3.8 & 2.9 & 0.7 & 1.3 \\
\hline Ingreso medio & 4.1 & 10.7 & 10.5 & 15.0 & 2.2 & 1.9 & 1.0 & 1.5 \\
\hline Ingreso alto & 5.6 & 16.1 & 16.0 & 23.1 & 2.3 & 2.1 & 1.1 & 1.6 \\
\hline Todos & 3.6 & 10.4 & 10.7 & 15.7 & 2.5 & 2.1 & 1.2 & 1.6 \\
\hline Clasificación de 1995 & 1940 & 1986 & 1995 & 2019 & $1940-1985$ & $1940-1994$ & $1986-2019$ & $1995-2019$ \\
\hline Ingreso bajo & 1.6 & 7.7 & 7.3 & 9.6 & 3.9 & 2.9 & 0.7 & 1.1 \\
\hline Ingreso medio & 3.5 & 10.4 & 10.7 & 15.7 & 2.7 & 2.2 & 1.2 & 1.6 \\
\hline Ingreso alto & 5.6 & 16.1 & 16.0 & 24.2 & 2.3 & 2.1 & 1.2 & 1.7 \\
\hline Todos & 3.6 & 10.4 & 10.7 & 15.7 & 2.5 & 2.1 & 1.2 & 1.6 \\
\hline
\end{tabular}

Nota: Los grupos de ingreso se construyeron con los terciles del PIB por habitante de 1986 y 1995; y, no se consideraron las entidades petroleras de Campeche y Tabasco

La figura 2 muestra la evolución de la mediana de cada grupo de ingreso obtenido a partir de los terciles en los que se dividieron las entidades federativas. En dicha figura se puede apreciar un mayor dinamismo de las entidades de ingreso bajo, principalmente el periodo que va de 1960 a 1981, el cual se desacelera de manera notable a partir de los años 1985 y 1986. Por otro lado, destaca el hecho de que el grupo de ingreso alto tiene un mayor crecimiento a partir de 1982 y que a pesar de tener pronunciadas fluctuaciones, la brecha que los separa de los grupos de ingreso medio e ingreso bajo tiende a ser mayor en este segundo periodo. 


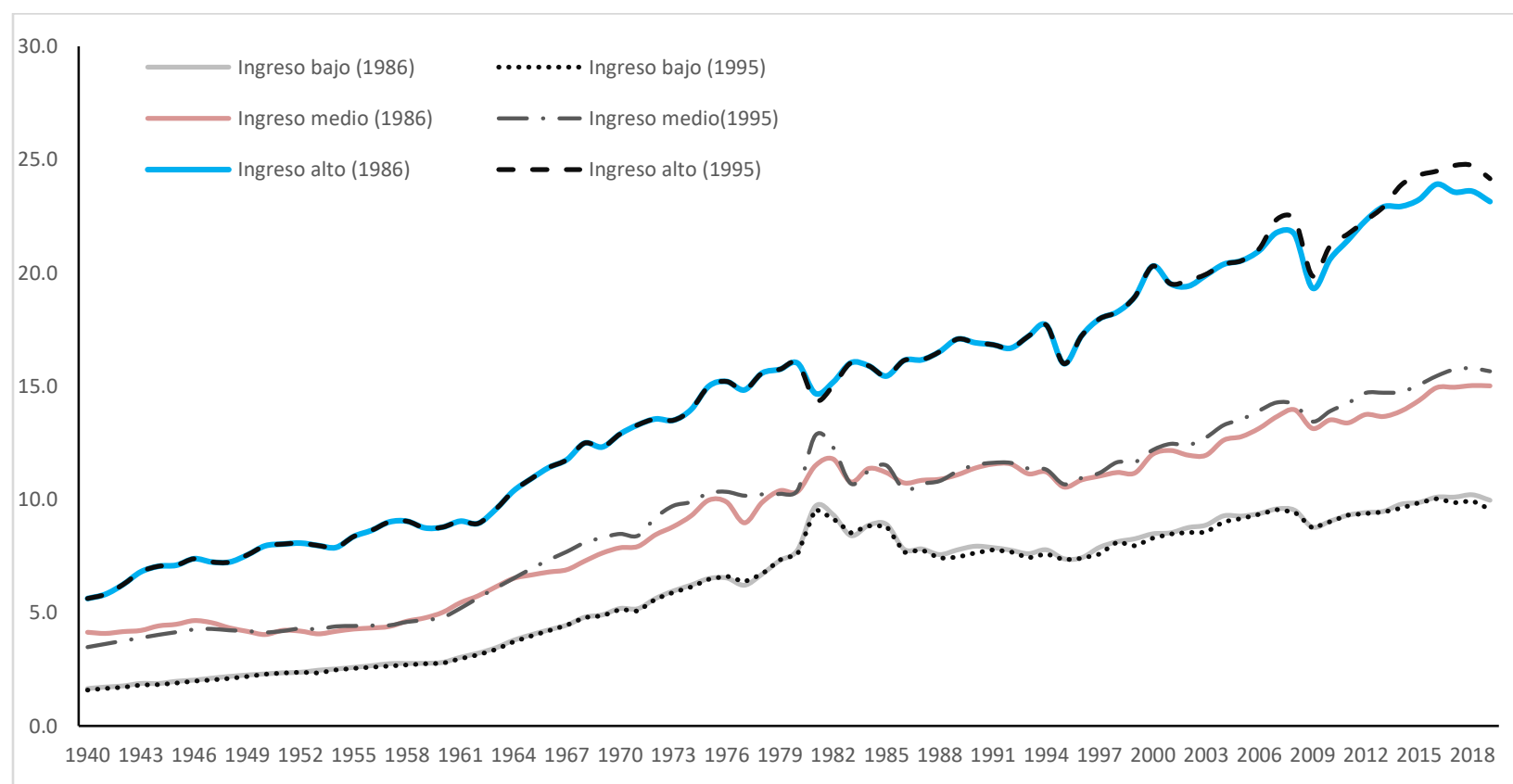

Figura 2. Tendencias de grupos de entidades por ingreso, clasificaciones 1986 y 1995

Miles de pesos de 1993

Nota: Los grupos de ingreso se construyeron con los terciles del PIB por habitante de 1986 y 1995; y, no se consideraron las entidades petroleras de Campeche y Tabasco

De igual forma, en la figura 2 se puede ver que el año de clasificación no marca una diferencia significativa en la evolución de la mediana para cada grupo de ingreso ya que prácticamente es el mismo.

En la figura 3 se muestra un análisis de la convergencia sigma tradicional del PIB por habitante por grupos de entidades por ingreso de acuerdo con las clasificaciones obtenidas para los años de 1986 y de 1995. En la figura 3 se puede ver que el comportamiento de sigma-convergencia muestran una forma de "U" para la totalidad de las entidades federativasen. Sin embargo, el comportamiento de la sigma-convergencia por grupos de ingreso muestra notables diferencias, lo cual es más notorio para los grupos de ingreso bajo ya que cuando se toma como año de referencia a 1986 el comportamiento de la convergencia sigma se asemeja al obtenido con la totalidad de las entidades federativas, mientras que cuando se toma como referencia el año de 1995 la sigmaconvergencia muestra una tendencia creciente en casi todo el periodo de análisis, con excecpción del periodo 1980-1995. Lo anterior sugiere que la falta de convergencia para el segundo periodo puede atribuirse principalmente al grupo de entidades de ingreso bajo principalmente a partir de 1995, un año después a la entrada en vigor del tratado de libre comercio de America del Norte. 


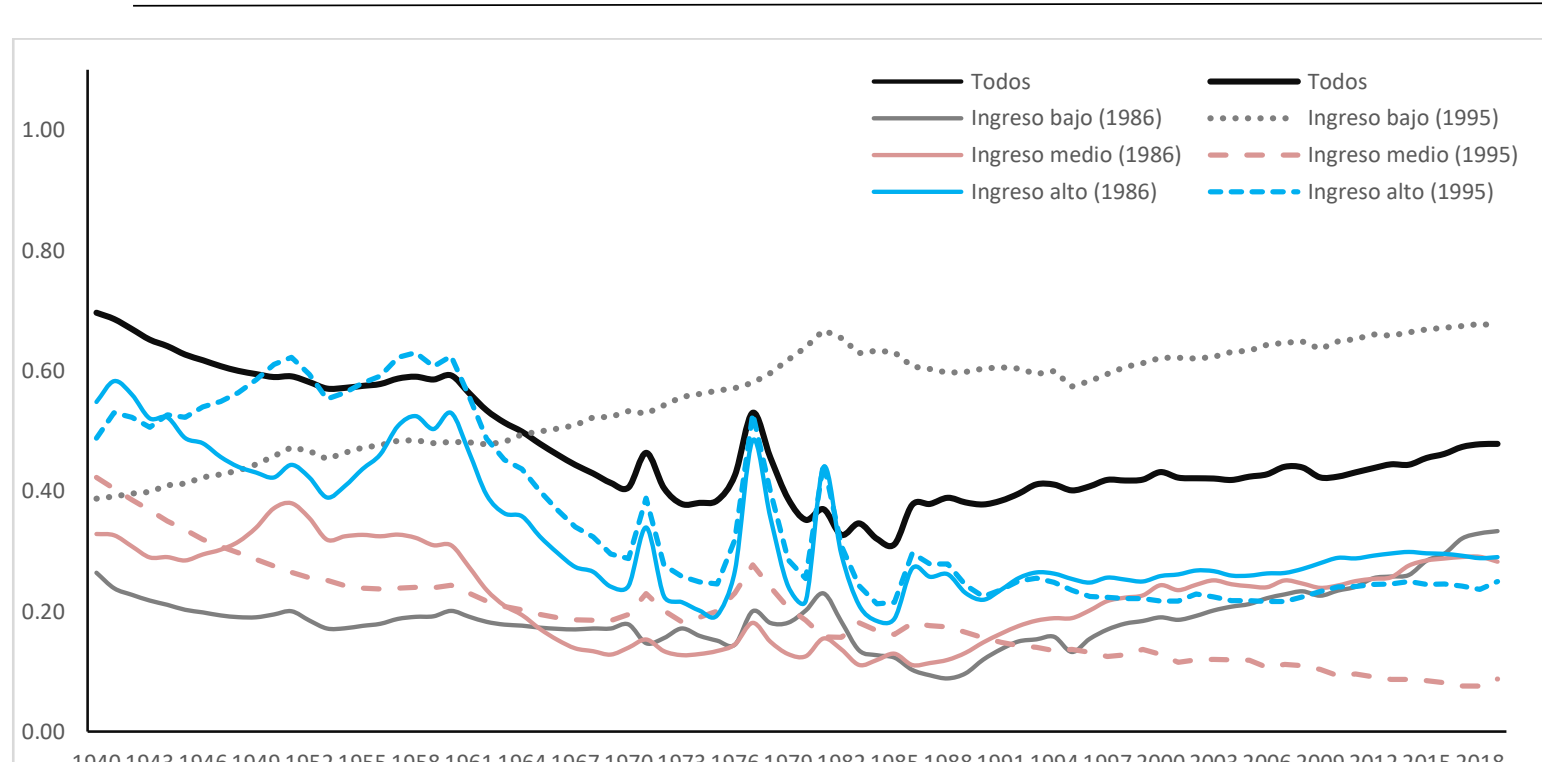

Figura 3. Convergencia sigma tradicional del PIB por habitante por grupos de entidades por ingreso, clasificaciones 1986 y 1995

Nota: La convergencia sigma se mide como la desviación estándar del logaritmo natural del PIB por habitante; la convergencia sigma para los grupos de ingreso considera los terciles del PIB por habitante de 1986 y 1995; $\mathrm{y}$, no se consideraron las entidades petroleras de Campeche y Tabasco

En tanto que la sigma-convergencia de los grupos de ingreso medio y alto muestra una tendencia decreciente principalmente la del grupo de ingreso medio de acuerdo a la clasificación efectuada con base al año de 1995. Estos hechos estilizados ponen de manifiesto la necesidad de efectuar pruebas formales de convergencia, como la que empleamos en este trabajo, a los grupos conformados por niveles de ingreso con el fin de determinar si algún grupo, o algunos grupos, pueden contribuir a la falta de convergencia en los resultados obtenidos con la totalidad de las entidades federativas analizadas.

\section{Metodología Econométrica}

El concepto de $\sigma$-convergencia es más conveniente cuando la variable en panel de interés, $y_{i t}$, cambia de signo con el tiempo, o bien, cuando el factor común $\theta_{t}$ puede contener o no tendencia estocástica.

El concepto $\sigma$-convergencia se define en términos de una disminución de la dispersión transversal a lo largo del tiempo. Este concepto fue sugerido originalmente por Hotelling (1933) y posteriormente definido de manera más formal por Kong, Phillips y Sul (2018). La convergencia relativa se centra en la $\sigma$-convergencia de las curvas de transición relativa (o coeficientes de ajuste de factores variables en el tiempo). Solo cuando $y_{i t}$ comparte el mismo promedio a largo plazo, la varianza transversal de $y_{i t}, K_{t}$, posiblemente converja a cero. De lo contrario, la varianza de la sección transversal de $y_{i t}$ converge en una constante distinta de cero en la medida que tanto $n$ y $T$ 
tienden a incrementarse considerablemente. Kong, Phillips y Sul (2018) llama a este tipo de $\sigma$ convergencia " $\sigma$-convergencia débil" y la definen estadísticamente como:

$$
\operatorname{Cov}\left(K_{t}, t\right) \leq 0
$$

La $\sigma$-convergencia débil es más restrictiva que la convergencia relativa, propuesta por Phillips y Sul (2007), cuando el factor común tiene un comportamiento de tendencia distinto. De acuerdo con Sul (2019), la diferencia entre la $\sigma$-convergencia débil y la convergencia relativa se puede mostrar si se considera que cada $y_{i t}$ tiene los siguientes componentes:

$$
y_{i t}=a_{i}+\left(b+\varepsilon_{i} t^{-\frac{1}{2}}\right) t+\eta_{i t} t^{-\beta}, \operatorname{con} \beta>0
$$

donde $\varepsilon_{i} \sim \operatorname{iid}\left(0, \sigma_{\varepsilon}^{2}\right)$ y $\eta_{i} \sim i i d\left(0, \sigma_{\eta}^{2}\right)$ y todos los términos $\left(a_{i}, \varepsilon_{i}, \eta_{i t}\right)$ son independientes. En lugar del término de tendencia lineal en (2) se puede incorporar un factor común estocástico. De acuerdo con Sul (2019), es posible mostrar que la convergencia relativa se mantiene, pero no la $\sigma$ convergencia débil tomando un par de elementos $\left(y_{i t}, y_{j t}\right)$, se tiene que:

$$
\operatorname{plim}_{t \rightarrow \infty} \frac{y_{i t}}{y_{j t}}=\operatorname{plim}_{t \rightarrow \infty} \frac{b+\varepsilon_{i} t^{-\frac{1}{2}}}{b+\varepsilon_{j} t^{-\frac{1}{2}}}=1
$$

pero al considerar las variaciones de la sección transversal, como $K_{n t}^{y}=n^{-1} \sum_{i=1}^{n}\left(y_{i t}-n^{-1} \sum_{i=1}^{n} y_{i t}\right)^{2}$, se tiene que:

$$
K_{t}^{y}=\operatorname{plim}_{n \rightarrow \infty} K_{n t}^{y}=\sigma_{a}^{2}+\sigma_{\varepsilon}^{2} t+\sigma_{\eta}^{2} t^{-2 \beta} \operatorname{con} \beta>0,
$$

donde $\sigma_{a}^{2}$ es la varianza de $a_{i}$. Siempre que $\sigma_{\varepsilon}^{2}>0$; la dispersión transversal de $y_{i t}$ se incrementa con el tiempo. Por tanto, la condición de que $\varepsilon_{i} \neq \varepsilon_{j}$ para algún $i \neq j$ es suficiente para que no se cumpla la $\sigma$-convergencia general.

Por otro lado, cuando el factor común contiene un componente de tendencia débil, la convergencia relativa no se mantiene, pero sí la $\sigma$-convergencia débil. Para ilustrarlo, Sul (2019) propone la siguiente especificación:

$$
y_{i t}=a_{i}+\varepsilon_{i} t^{-\frac{1}{2}}+\eta_{i t} t^{-\beta}, \operatorname{con} \beta>0
$$


En este caso, la convergencia relativa no se mantiene porque:

$$
\operatorname{plim}_{t \rightarrow \infty} \frac{y_{i t}}{y_{j t}}=\operatorname{plim}_{t \rightarrow \infty} \frac{a_{i}+\varepsilon_{i} t^{-\frac{1}{2}}+\eta_{i t} t^{-\beta}}{a_{i}+\varepsilon_{j} t^{-\frac{1}{2}}+\eta_{j t} t^{-\beta}}=\frac{a_{i}}{a_{j}} \neq 1 .
$$

Pero la $\sigma$-convergencia débil se mantiene ya que:

$$
K_{t}^{y}=\sigma_{a}^{2}+\sigma_{\varepsilon}^{2} t^{-1}+\sigma_{\eta}^{2} t^{-2 \beta} \rightarrow \sigma_{a}^{2}
$$

Por lo tanto, la noción de $\sigma$-convergencia débil es de aplicación más general, pero también más restrictiva que la convergencia relativa cuando los factores comunes tienen tendencias estocásticas distintas.

Kong, Phillips y Sul (2018) proponen la siguiente prueba de $\sigma$-convergencia débil. Sea $K_{n t}^{y}$ la varianza muestral transversal de $y_{i t}$. Posteriormente, se construye el estadístico $t$ de la estimación de MCO de $\widehat{\phi}$ basado en el estimador HAC de Newey-West con $L=\operatorname{int}\left(T^{\frac{1}{3}}\right)$ de la siguiente regresión de tendencia simple:

$$
\begin{gathered}
K_{n t}^{y}=a+\phi t+u_{t} \\
t_{\widehat{\phi}_{n t}}=\frac{\phi}{\sqrt{\widehat{\Omega}_{u}^{2} / \sum_{t=1}^{T}\left(t-T^{-1} \sum_{t=1}^{T} t\right)^{2}}}
\end{gathered}
$$

Dónde $\widehat{u}_{t}=K_{n t}^{y}-\widehat{a}-\widehat{\phi} t, \mathrm{y}$

$$
\widehat{\Omega}_{u}^{2}=\frac{1}{t} \sum_{t=1}^{T} \widehat{u}_{t}^{2}+2 \frac{1}{t} \sum_{l=1}^{L} \vartheta_{l L} \sum_{t=1}^{T-l} \widehat{u}_{t} \widehat{u}_{t+l}
$$

y $\vartheta_{l L}$ es el núcleo de pesos de rezagos de Bartlett. Entonces la relación $t$ tiene los siguientes límites;

$$
\begin{array}{lr}
t_{\widehat{\phi}}<-\sqrt{3} \text { o } t_{\widehat{\phi}} \rightarrow-\infty & \text { si } y_{i t} \text { es } \sigma \text {-convergencia débil. } \\
t_{\widehat{\phi}}<-\sqrt{3} \text { o } t_{\widehat{\phi}} \rightarrow-\infty & \text { si } y_{i t} \text { es divergente. } \\
t_{\hat{\phi}}<-\sqrt{3} \text { o } t_{\widehat{\phi}} \rightarrow-\infty & \text { si } y_{i t} \text { es fluctuante. }
\end{array}
$$


Es necesario tener en cuenta que $\sqrt{3} \simeq 1.73$, por lo que Kong, Phillips y Sul (2018) sugieren que el valor crítico habitual del $5 \%$ (prueba de un lado, 1,65) se puede utilizar para probar la $\sigma$ convergencia débil. Es decir, dependiendo de la relación $t$, se puede comprobar si $y_{i t}$ es $\sigma$ convergencia débil., fluctuante o divergente como se muestra en el cuadro 3 :

Cuadro 3. Resultado de la prueba $\sigma$-convergencia débil de acuerdo con el valor del estadístico $t$.

\begin{tabular}{|c|c|c|c|}
\hline Valor del estadístico: & $t_{\hat{\phi}}<-1.65$ & $-1.65<t_{\hat{\phi}}<1.65$ & $-1.65<t_{\hat{\phi}}$ \\
\hline Resultado: & $\sigma$-convergencia débil & fluctuante & $\sigma$-divergencia \\
\hline
\end{tabular}

Fuente: Adaptado de Sul (2019).

Sul (2019) sostiene que la regresión de tendencia en (8) está incorrectamente especificada a menos que $y_{i t}$ esté fluctuando con el tiempo (en este caso, $\phi=0$ ). Además, en la medida que $n$ y $T$, tiendan a ser infinitamente grandes la estimación de MCO de $\phi$ converge a cero, pero su razón $t$ es divergente, crece o decrece infinitamente, si $y_{i t}$ diverge (o hay $\sigma$-convergencia débil). En otras palabras, incluso cuando $\widehat{t}<1.73$; no significa que $K_{n t}^{y}$ divergerá infinitamente de manera negativa a largo plazo.

\section{Resultados}

Los resultados de la prueba de $\sigma$-convergencia débil aplicadas al logaritmo natural del PIB per cápita, tanto a la totalidad de las entidades federativas como cuando se excluyen los estados petroleros de Campeche y de Tabasco, se presentan en los cuadros 4 y 5, respectivamente. La prueba se realizó para todo el periodo de analisis, el cual comprende de 1940 a 2019, y para los periodos en los que suele dividirse la muestra tomando tanto a 1985 como a 1995 como los años de quiebre, de los cuales el primero corresponde al año en que suele ubicarse el fin del periodo de sustitución de importaciones en México, mientras que el segundo al año en el que entra en vigor el tratado de libre comercio de América del Norte, siendo este último de interés para evaluar si la mayor liberalización promovida por el tratado de libre comercio de América del Norte promovió o no una mayor convergencia a nivel de regiones en México. Las pruebas también se efectuaron a los grupos de ingreso bajo, medio y alto que se obtuvieron de clasificar a las entidades federativas de acuerdo con los terciles tomando como referencia los años 1985 y 1995. 
Cuadro 4. Resultados de la prueba sigma-convergencia débil tomando a 1995 como el año de quiebre.

\begin{tabular}{|c|c|c|c|c|c|c|c|c|}
\hline \multicolumn{8}{|c|}{ Todos los estados } \\
\hline \multicolumn{3}{|c|}{ De 1940 a 1994 } & \multicolumn{3}{c|}{$1995-2019$} & \multicolumn{3}{c|}{ Todo el periodo } \\
\hline$\hat{\phi}_{n t} \times 100$ & $\hat{t}_{\phi_{n t}}(3)$ & $\hat{t}_{\phi_{n t}}(4)$ & $\hat{\phi}_{n t} \times 100$ & $\hat{t}_{\phi_{n t}}(2)$ & $\hat{t}_{\phi_{n t}}(3)$ & $\hat{\phi}_{n t} \times 100$ & $\hat{t}_{\phi_{n t}}(4)$ & $\hat{t}_{\phi_{n t}}(5)$ \\
\hline-0.4814 & -7.0167 & -6.5518 & 0.1791 & 4.0280 & 3.7587 & -0.2543 & -4.8818 & -4.5710 \\
\hline \multicolumn{8}{|c|}{ Excluyendo a las entidades petroleras (Campeche y Tabasco) } \\
\hline \multicolumn{3}{|c|}{ De 1940 a 1994-2019 } & \multicolumn{3}{|c|}{ Todo el periodo } \\
\hline$\hat{\phi}_{n t} \times 100$ & $\hat{t}_{\phi_{n t}}(3)$ & $\hat{t}_{\phi_{n t}}(4)$ & $\hat{\phi}_{n t} \times 100$ & $\hat{t}_{\phi_{n t}}(2)$ & $\hat{t}_{\phi_{n t}}(3)$ & $\hat{\phi}_{n t} \times 100$ & $\hat{t}_{\phi_{n t}}(4)$ & $\hat{t}_{\phi_{n t}}(5)$ \\
\hline-0.6310 & -10.950 & -10.351 & 0.2235 & 6.4274 & 6.2424 & -0.2975 & -4.6288 & -4.2988 \\
\hline
\end{tabular}

Notas: Los números entre paréntesis son los rezagos incorporados en el cálculo de la varianza de Newey-West.

Cuadro 5. Resultados de la prueba sigma-convergencia débil tomando a 1985 como el año de quiebre.

\begin{tabular}{|c|c|c|c|c|c|}
\hline \multicolumn{6}{|c|}{ Todos los estados } \\
\hline \multicolumn{3}{|c|}{ De 1940 a 1984} & \multicolumn{3}{|c|}{ De 1985 a 2019} \\
\hline$\hat{\phi}_{n t} \times 100$ & $\hat{t}_{\phi_{n t}}(3)$ & $\hat{t}_{\phi_{n t}}(4)$ & $\hat{\phi}_{n t} \times 100$ & $\hat{t}_{\phi_{n t}}(3)$ & $\hat{t}_{\phi_{n t}}(4)$ \\
\hline-0.6583 & -9.6985 & -9.2862 & 0.0284 & 0.5437 & 0.5220 \\
\hline \multicolumn{6}{|c|}{ Excluyendo a las entidades petroleras (Campeche y Tabasco) } \\
\hline \multicolumn{3}{|c|}{ De 1940 a 1984} & \multicolumn{3}{|c|}{ De 1985 a 2019} \\
\hline$\hat{\phi}_{n t} \times 100$ & $\hat{t}_{\phi_{n t}}(3)$ & $\hat{t}_{\phi_{n t}}(4)$ & $\hat{\phi}_{n t} \times 100$ & $\hat{t}_{\phi_{n t}}(3)$ & $\hat{t}_{\phi_{n t}}(4)$ \\
\hline-0.7799 & -13.0816 & -12.6338 & 0.2434 & 10.8423 & 10.5404 \\
\hline
\end{tabular}

Notas: Los números entre paréntesis son los rezagos incorporados en el cálculo de la varianza de Newey-West.

Los resultados de los cuadros 4 y 5 muestran que independientemente del año en los que suele dividirse la muestra, únicamente se encuentra evidencia de convergencia en el primer periodo de análisis tanto para la totalidad de los estados como cuando se excluyen las entidades federativas de Campeche y Tabasco, que son consideradas como petroleras. Los resultados de la prueba sigmaconvergencia débil también revelan convergencia para todo el periodo con ambas muestras, lo cual sugiere que en términos generales la dispersión del PIB per cápita se ha reducido a pesar de tener ese comportamiento en forma de "U" descrito en la sección 3 de los datos.En tanto que los resultados de la prueba para el segundo periodo son heterogéneos ya que revelan evidencia de divergencia para ambas muestras, con la totalidad de las entidades y cuando se excluyen las petroleras, tomando como año de quiebre a 1995. Este mismo resultado se obtiene cuando se considera como año de quiebre a 1985 y se excluyen las entidades petroleras, no obstante, la prueba efectuada para la totalidad de los estados sugiere que en el periodo que va de 1985 a 2019 la sigma convergencia es fluctuante.

De esta manera no existe evidencia de $\sigma$-convergencia débil para el segundo periodo de análisis caracterizado principalmente por una mayor liberalización comercial caracterizado principalmente por la entrada en vigor del tratado de libre comercio. Por el contrario existe evidencia de divergencia en este periodo, lo cual sugiere un posible efecto diferenciado en las regiones como consecuencia de la mayor apertura comercial que ha promovido y alentado que algunas regiones 
crezcan a un mayor ritmo y a otras que no se encuentran lo suficientemente ancladas a este dinamismo se han ralentizado.

El cuadro 6 muestra los resultados de las pruebas de sigma convergencia débil aplicadas a los grupos de estados clasificados de acuerdo a los terciles como de ingreso bajo, medio y alto, tanto para todo el periodo como por periodos tomando como año de quiebre a $1995^{3}$. Como se puede ver en dicho cuadro, hay evidencia de sigma convergencia débil en todos los grupos de ingreso en el primer periodo y en todo el periodo bajo estudio. No obstante, para el segundo periodo los resultados no son tan homogéneos ya que para los grupos de estados de bajo y alto ingreso el resultado de la prueba sugiere divergencia. Con fines de comparación mostramos los resultados de aplicar las pruebas a estos grupos de entidades tomando como fecha de quiebre el año de 1986, que representa el año en que culmina el periodo de sustitución de importaciones y en que México ingresó al GATT, cuyos resultados se presentan en el cuadro 7. Los resultados son muy similares, con excepción de los obtenidos para los de ingreso medio en el segundo periodo, de 1986 a 2019, y para todo el periodo en su conjunto, ya que para el segundo periodo no se encuentra evidencia de convergencia-débil para las entidades que conforman dicho grupo, mientras que para todo el periodo se encuentra evidencia de que el indicador es fluctuante. Otra discrepancia de los resultados obtenidos tomando como año de quiebre a 1986 es el que se obtiene para las entidades de ingreso bajo ya que en este caso hay evidencia de que dicho indicador es fluctuante mientras que cuando el año de quiebre y de clasificación es 1995 se obtiene evidencia de convergencia.

Cuadro 6. Resultados de la prueba tomando a 1995 como año de quiebre y de clasificación para los distintos grupos de ingreso.

\begin{tabular}{|c|c|c|c|c|c|c|c|c|}
\hline \multirow{2}{*}{\multicolumn{3}{|c|}{$1970-1994$}} & \multicolumn{3}{|c|}{ Ingreso alto } & & & \\
\hline & & & \multicolumn{3}{|c|}{$1995-2019$} & \multicolumn{3}{|c|}{ Todo el periodo } \\
\hline$\hat{\phi}_{n t} \times 100$ & $\hat{t}_{\phi_{n t}}(3)$ & $\hat{t}_{\phi_{n t}}(4)$ & $\hat{\phi}_{n t} \times 100$ & $\hat{t}_{\phi_{n t}}(2)$ & $\hat{t}_{\phi_{n t}}(3)$ & $\hat{\phi}_{n t} \times 100$ & $\hat{t}_{\phi_{n t}}(4)$ & $\hat{t}_{\phi_{n t}}(5)$ \\
\hline-0.6135 & -6.0435 & -5.6035 & 0.0557 & 3.7534 & 3.4925 & -0.4215 & -6.6257 & -6.1956 \\
\hline & & & \multicolumn{3}{|c|}{$\begin{array}{l}\text { Ingreso medio } \\
\end{array}$} & & & \\
\hline \multicolumn{3}{|c|}{ 1940-1994 } & \multicolumn{3}{|c|}{$1995-2019$} & \multicolumn{3}{|c|}{ Todo el periodo } \\
\hline$\hat{\phi}_{n t} \times 100$ & $\hat{t}_{\phi_{n t}}(3)$ & $\hat{t}_{\phi_{n t}}(4)$ & $\hat{\phi}_{n t} \times 100$ & $\hat{t}_{\phi_{n t}}(2)$ & $\hat{t}_{\phi_{n t}}(3)$ & $\hat{\phi}_{n t} \times 100$ & $\hat{t}_{\phi_{n t}}(4)$ & $\hat{t}_{\phi_{n t}}(5)$ \\
\hline-0.1894 & -6.0836 & -5.6435 & -0.0543 & -19.0265 & -20.4421 & -0.1360 & -7.5955 & -7.1675 \\
\hline & & & \multicolumn{3}{|c|}{ Ingreso bajo } & & & \\
\hline \multicolumn{3}{|c|}{ 1940-1994 } & \multicolumn{3}{|c|}{$1995-2019$} & \multicolumn{3}{|c|}{ Todo el periodo } \\
\hline$\hat{\phi}_{n t} \times 100$ & $\hat{t}_{\phi_{n t}}(3)$ & $\hat{t}_{\phi_{n t}}(4)$ & $\hat{\phi}_{n t} \times 100$ & $\hat{t}_{\phi_{n t}}(2)$ & $\hat{t}_{\phi_{n t}}(3)$ & $\hat{\phi}_{n t} \times 100$ & $\hat{t}_{\phi_{n t}}(4)$ & $\hat{t}_{\phi_{n t}}(5)$ \\
\hline-0.2974 & -9.8894 & -9.0711 & 0.1691 & 9.7058 & 9.1343 & -0.1744 & -6.2425 & -5.7816 \\
\hline
\end{tabular}

Notas: Con 30 estados excluyendo Campeche y Tabasco. Los números son los rezagos incorporados en el cálculo de la varianza de Newey-West.

${ }^{3}$ En la obtención de los terciles se excluyeron las entidades de Campeche y Tabasco. 
Cuadro 7. Resultados de la prueba tomando a 1986 como año de quiebre y de clasificación para los distintos grupos de ingreso.

\begin{tabular}{|c|c|c|c|c|c|c|c|c|}
\hline \multirow{2}{*}{\multicolumn{3}{|c|}{$1940-1985$}} & \multicolumn{3}{|c|}{ Ingreso alto } & & & \\
\hline & & & \multicolumn{3}{|c|}{$1986-2019$} & \multicolumn{3}{|c|}{ Todo el periodo } \\
\hline$\hat{\phi}_{n t} \times 100$ & $\hat{t}_{\phi_{n t}}(3)$ & $\hat{t}_{\phi_{n t}}(4)$ & $\hat{\phi}_{n t} \times 100$ & $\hat{t}_{\phi_{n t}}(2)$ & $\hat{t}_{\phi_{n t}}(3)$ & $\hat{\phi}_{n t} \times 100$ & $\hat{t}_{\phi_{n t}}(4)$ & $\hat{t}_{\phi_{n t}}(5)$ \\
\hline-0.5292 & -5.8217 & -5.5162 & 0.0878 & 6.0726 & 5.9856 & -0.0025 & -5.1436 & -4.8555 \\
\hline & & & \multicolumn{3}{|c|}{ Ingreso medio } & & & \\
\hline \multicolumn{3}{|c|}{$1940-1985$} & \multicolumn{3}{|c|}{ 1986-2019 } & \multicolumn{3}{|c|}{ Todo el periodo } \\
\hline$\hat{\phi}_{n t} \times 100$ & $\hat{t}_{\phi_{n t}}(3)$ & $\hat{t}_{\phi_{n t}}(4)$ & $\hat{\phi}_{n t} \times 100$ & $\hat{t}_{\phi_{n t}}(2)$ & $\hat{t}_{\phi_{n t}}(3)$ & $\hat{\phi}_{n t} \times 100$ & $\hat{t}_{\phi_{n t}}(4)$ & $\hat{t}_{\phi_{n t}}(5)$ \\
\hline-0.0027 & -6.0242 & -5.5740 & 0.0021 & 7.8066 & 11.7231 & -0.0522 & -1.5471 & -1.4300 \\
\hline & & & \multicolumn{3}{|c|}{ Ingreso bajo } & & & \\
\hline \multicolumn{3}{|c|}{$1940-1985$} & \multicolumn{3}{|c|}{ 1986-2019 } & \multicolumn{3}{|c|}{ Todo el periodo } \\
\hline$\hat{\phi}_{n t} \times 100$ & $\hat{t}_{\phi_{n t}}(3)$ & $\hat{t}_{\phi_{n t}}(4)$ & $\hat{\phi}_{n t} \times 100$ & $\hat{t}_{\phi_{n t}}(2)$ & $\hat{t}_{\phi_{n t}}(3)$ & $\hat{\phi}_{n t} \times 100$ & $\hat{t}_{\phi_{n t}}(4)$ & $\hat{t}_{\phi_{n t}}(5)$ \\
\hline-0.2974 & -9.8894 & -9.0711 & 0.1691 & 9.7058 & 9.1343 & 0.0310 & 1.7573 & 1.6453 \\
\hline
\end{tabular}

Notas: Con 30 estados excluyendo Campeche y Tabasco. Los números son los rezagos incorporados en el cálculo de la varianza de Newey-West.

Las pruebas efectuadas a las entidades clasificadas por grupos de ingresos y para distintos periodos permiten identificar tanto los grupos y en que periodos de tiempo se cumple o no la hipótesis de convergencia. De esta manera, en el periodo posterior a la entrada en vigor del TLCAN las entidades correspondientes a los grupos de ingreso bajo y alto divergen, mientras que para el primer periodo todos los grupos de ingreso convergen al igual que para todo el periodo en su conjunto. Otro resultado importante a destacar es que las entidades de ingreso medio reportan convergencia tanto para todo el periodo como en los periodos en que se dividió la muestra.

Por otro lado, cuando tanto la clasificación de los grupos así como su periodización se realiza con base en el PIB per cápita del año de 1986, se encuentra convergencia en el primer periodo que va de 1940 a 1985 para los tres grupos de ingreso así como también para los de ingreso alto para todo el periodo, además que este indicador es fluctuante para los de ingreso medio y bajo para todo el periodo y divergencia para todos los grupos de ingreso en el segundo periodo que va de 1986 a 2019.

A grandes rasgos, los resultados obtenidos con sigma-convergencia, presentados en la sección 3, son consistentes con los obtenidos con la prueba sigmaconvergencia débil , sólo que en este caso, la prueba sigma convergencia débil es una prueba formal que permite identificar si una variable de un conjunto de regiones converge, fluctua o diverge a diferencia del análisis de sigmaconvergencia tradicional que es más informal y de apreciación subjetiva. 


\section{Conclusiones}

En este trabajo se analiza la hipótesis de convergencia a nivel de entidades federativas de la República Mexicana en el periodo 1940 a 2019. El analisis de la convergencia es relevante dados los altos niveles de desigualdad registrados en las últimas décadas en México, además de los notables cambios socioeconómicos, políticos y en materia comercial experimentados durante el periodo de analisis, Mendoza et al. (2020).

Al someter a prueba la hipótesis de convergencia bajo el enfoque de $\sigma$-convergencia débil recientemente propuesto por Kong, Phillips y Sul (2019), se encuentra evidencia de $\sigma$-convergencia débil a nivel de entidades federativas para México en el primer periodo en el que suele dividirse la muestra, ya sea en 1986 o hasta 1994, para la totalidad de las entidades federativas, cuando se excluyen los estados pretroleros, e incluso por grupos de ingreso independientemente del año que se elija para efectuar la clasificación. Sin embargo, las pruebas no revelan evidencia de $\sigma$ convergencia débil en el periodo de liberalización comercial, por el contrario existe evidencia de divergencia para este periodo, con excepción de que para el caso que incluye la totalidad de los estados estados se encontró un comportamiento fluctuante en este periodo y de convergencia únicamente para las entidades de ingreso medio cuando la clasificación de los grupos de ingreso se realiza en base al año de 1995.

Estos resultados tienden a reforzar la idea de que los efectos de la apertura comercial han tenido un efecto diferenciado en las regiones de México, no obstante se requiere una mayor investigación al respecto en la que se realicen pruebas con mayores niveles de desagregación o en grupos que se pueden conformar en base a distintos criterios, lo cual contribuirá a identificar si la ausencia de convergencia encontrada en todas las unidades es atribuible al comportamiento de ciertos grupos.

\section{Referencias}

[1] Aguilar-Retureta, José 2016. Regional Income Distribution in Mexico: New Long-Term Evidence, 18952010. Economic History of Developing Regions, Vol. 31, Issue. 2-3, p. 225.

[2] Apergis, N., C. Christou y S. Miller. 2012. Convergence Patterns in Financial Development: Evidence from Club Convergence. Empirical Economics, 43: 1011-1040.

[3] Bai J. y Ng S. (2002). Determining the Number of Factors in Approximate Factor Models. Econometrica, 70, 191-221.

[4] Barro, R.J., y X. Sala-i-Martin (1990). Economic growth and convergence across the United States. NBER working paper, 3419. National Bureau of Economic Research, Cambridge.

[5] Barro, R.J., y X, Sala-i-Martin (1992). Convergence. Journal of Political Economy, 100 (2): 223-251.

[6] Barro, R. J. y X. Sala-i-Martin (2004). Economic growth, 2nd ed., London: MIT Press.

[7] Becerril. O. I. Alvarez, L. Del Moral (2010). Do infrastructures influence the convergence of efficiency in Mexico?, Journal of Policy Modeling, 32 pp. 120-137

[8] Brock, G. y Germán-Soto, V, 2013. Regional Industrial Growth in Mexico: Do Human Capital and Infrastructure Matter?, Journal of Policy Modeling, 35, 228-242. 
[9] Brock, G. (2019). The real Oaxaca decomposition: convergence within Mexico's Oaxaca region in the twenty-first century-Do types of crime and religious belief matter?. Economic Change and Restructuring, https://doi.org/10.1007/s10644-019-09255-7

[10] Cabrera, C. L. F. (2002). Convergence and Regional Economic Growth in Mexico: 1970-1995. MPRA Paper Núm. 4026. Disponible en: http://mpra.ub.uni muenchen.de/4026/

[11] Carrion-I-Silvestre, J. y German-Soto, V. (2007). Stochastic convergence amongst Mexican states. Regional Studies, 41 (4), 531-541.

[12] Carrion-I-Silvestre, J. y German-Soto, V. (2009). Panel Data Stochastic Convergence Analysis of the Mexican Regions. Empirical Economics, 37 (2), pp. 303-327.

[13] Chiquiar, Daniel (2005). Why Mexico's regional income convergence broke down. Journal of Development Economics, Volume 77, Issue 1, pp. 257-275.

[14] Díaz-Dapena A., Fernández-Vázquez E., Garduño-Rivera R. y Rubiera-Morollon F. (2019). Economic integration and regional convergence: effects of NAFTA on local convergence in Mexico, 1980-2008. Appl Econ, 1-13

[15] Esquivel, G. (1999). Convergencia Regional en México, 1940-1995. El Trimestre Económico, LXVI, 264, 725-761.

[16] Esquivel, G. (1999). Convergencia regional en México, 1940-1995. El Trimestre Económico, 66(4): 725761.

[17] Esquivel, G. y Messmacher, M. (2002). Sources of Regional (non) Convergence in Mexico. Paper presented at the Wider/Cornell/LSE Mexico Conference on Spatial Inequality in Latin America. Institute of Public Policy and Development Studies, Universidad de las Americas, Puebla.

[18] Friedman, M. (1992). Do old fallacies ever die?. Journal of Economic Literature, 30(4), 2129-2132.

[19] Fonseca, F., Llamosas, I y Rangel, E. (2018). Liberalización económica e impactos externos. La hipótesis de convergencia para las entidades federativas en México, 1994 - 2015. Documentos de Investigación, Working Papers, No. 2018-26, Banco de México.

[20] García Verdú, R. (2002). Income dynamics across states in Mexico: 1940-2000, Banco de México, Unpublished manuscript

[21] German-Soto, V. (2005). Generacion del producto interno bruto mexicano por entidad federativa, 1940-1992. El Trimestre Económico, vol. LXXII (3), num. 287, pp. 617-653.

[22] Germán-Soto, V. y Escobedo, J. (2011). ¿Ha ampliado la liberalización comercial la desigualdad económica entre los estados mexicanos? Un análisis desde la perspectiva econométrico-espacial. Economía Mexicana, Nueva Época, XX, 1, pp. 37-77.

[23] German-Soto, Vicente (2014). Mexican Population by federal entity 1940-2020, Saltillo: own estimates.

[24] German-Soto, V., Rodríguez Pérez, R., \& Gallegos Morales, A. (2020). Exposure to globalization and regional convergence in Mexico. Estudios Económicos, 35(2), 267-295. https://doi.org/10.24201/ee.v35i2.404

[25] Gómez, Manuel y Daniel Ventosa-Santaulària (2008). Liberalización comercial y convergencia regional de ingreso en México. El Trimestre Económico, LXXVI (1), Fondo de Cultura Económica, México, pp. 215-235.

[26] Gómez, M., y Ventosa-Santaularia, D. (2009). Liberación comercial y convergencia regional del ingreso en México. El Trimestre Económico, 76(301), 215-235.

[27] Gómez-Zaldívar, M. y D. Ventosa-Santaulària (2010). Per Capita Output Convergence: The DickeyFuller Test Under the Simultaneous Presence of Stochastic and Deterministic Trends. Annales d'Economie et de Statistique, No. 99/100, 429-445.

[28] Gómez-Zaldívar, M. y Ventosa-Santauràlia, D. (2012). Regional output convergence in Mexico. Latin America Journal of Economics, 49 (2), 217-236. 
REMEF (The Mexican Journal of Economics and Finance)

La hipótesis de convergencia en México: un enfoque de $\sigma$-convergencia débil

[29] Hotelling, H. (1933). Review of the triumph of mediocrity in business by Horace Secrist. Journal of the American Statistical Association, 28, 463-465.

[30] Jangam, B.P., y Akram, V., (2019). Do Prices Converge Among Indonesian Cities? an Empirical Analysis, Bulletin of Monetary Economics and Banking, 22: $239 \quad-\quad 262$. https://doi.org/10.21098/bemp.v22i3.1152

[31] Johnson, Paul y Chris Papageorgiou (2020). What Remains of Cross-Country Convergence?. Journal of Economic Literature, 58 (1): 129-75. DOI: 10.1257/jel.20181207

[32] Jordaan, J. y Sanchez-Reaza, J, 2006. Trade Liberalization and Location: Empirical Evidence for Mexican Manufacturing Industries 1980-2003. Review of Regional Studies, 36, 279-303.

[33] Kido-Cruz, A. y M.T. Kido-Cruz. 2015. Convergencia económica en la región sur de México: un análisis municipal durante el periodo 1990-2010, Economía, Sociedad y Territorio, 15(49): 697-722.

[34] Kong, J., Phillips, P. C. B., \& Sul, D. (2019). Weak $\sigma$-convergence: Theory and applications. Journal of Econometrics, 209, 185-207.

[35] Kong, J., Phillips, P.C.B. and Sul, D. (2020). Testing Convergence Using HAR Inference, Li, T., Pesaran, M.H. and Terrell, D. (Ed.) Essays in Honor of Cheng Hsiao (Advances in Econometrics, Vol. 41), Emerald Publishing Limited, pp. 25-72. https://doi.org/10.1108/S0731-905320200000041002

[36] Lucas, R. (1988). On the Mechanics of Economic Development, Journal of Monetary Economics, 22, 342.

[37] Mendoza-Velázquez, Alfonso; Daniel Ventosa-Santaulària and Vicente German-Soto. (2019). Mexico's inter-regional inequality: a convergent process?, Empirical Economics, Vol. 56 Iss. 5 (2019) p. 1683 1705 ISSN: 0377-7332 Disponible en: http://works.bepress.com/vicente_german_soto/89/

[38] Mallick, R. y Carayannis, E. (1994). Regional economic convergence in Mexico: an analysis by industry, Growth and Change, 25: 325-334

[39] Mendoza, M. A. (2014). Metodología de interpolación estructural-espacial para la generación de una serie continua del pib y población por entidad federativa: 1970-2012, Documento de trabajo, UNAM, Facultad de Economía.

[40] Mendoza-Velázquez, A., Ventosa-Santaulària, D. y Germán-Soto, V. (2019). Mexico's inter-regional inequality: a convergent process?. Empirical Economics, 56, 1683-1705 https://doi.org/10.1007/s00181-017-1401-8

[41] Mendoza-Velázquez, A.; Vicente German-Soto, Mercedes Monfort y Javier Ordóñez. (2020). Club convergence and inter-regional inequality in Mexico, 1940-2015, Applied Economics, 52:6, 598-608, doi: 10.1080/00036846.2019.1659491

[42] Messmacher, M., 2000. Desigualdad Regional en México. El Efecto del TLCAN y Otras Reformas Estructurales. Documento de Investigación 2000-4, Banco de México.

[43] Narayan, Laxmi (2017). Convergence or divergence: An analysis of regional disparities in South Asian countries. International Journal of Multidisciplinary Research and Development, 4(7): 436-443.

[44] Plata, L., J. Calderón y S. Salazar. 2013. Convergencia económica en México 1950-2003, Perspectivas. Revista de Análisis de Economía, Comercio y Negocios Internacionales, pp. 141-168.

[45] Diaz, J., A. Sánchez, y M. Á. Mendoza. 2009. Convergencia hacia la economía regional líder en México: un análisis de cointegración en panel. El Trimestre Económico, 76(302(2)): 407-431. https://doi.org/10.20430/ete.v76i302.532

[46] Phillips, P. C. B. y Sul, D. (2007). Transition modelling and econometric convergence tests, Econometrica, 75, 1771-1855.

[47] Ramón, H. Juan y Rivera Bátiz, L. (1996). Regional Growth in Mexico: 1970-1993. IMF Working Paper, 96/92. 
[48] Rodríguez Benavides, D., M.A. Mendoza y F. Venegas Martínez. (2016a). ¿Realmente existe convergencia regional en México? Un modelo de datos-panel TAR no lineal, Economía, Sociedad y Territorio, 16(50): 197-227.

[49] Rodríguez Benavides, D., F. López y M.A. Mendoza. (2016b). Clubs de convergencia regional en México: un análisis a través de un modelo no lineal de un solo factor, Investigaciones Regionales - Journal of Regional Research, 34: 7-22.

[50] Rodríguez Benavides, D., J. C. Trejo García, and M. Á. Mendoza González. (2016c). Pair-Wise Approach to Test the Regional Convergence Hypothesis in Mexico. Journal of Reviews on Global Economics 5: 5968.

[51] Rodríguez-Gámez, L. I., y Cabrera-Pereyra, J. A. (2019). Convergencia municipal en México con modelos de econometría espacial (1999 - 2014). EconoQuantum, 16(1), 7-32.

[52] Rodríguez-Gámez, L. I., y Cabrera-Pereyra, J. A. (2020). Convergencia económica entre municipios mexicanos: un enfoque de parámetros locales. Ensayos Revista de Economía, 39 (2), 143-186, Noviembre, DOI: http://dx.doi.org/10.29105/ensayos39.2-2.

[53] Rodríguez Oreggia, E., 2001. Regional Policies and Income Disparities in Mexico: A Review of Convergence Aspects. CEIDIR'S REVIEW: Electronic Publication on Local Economic Development. Available at http://orbita.starmedia.com/ delenlace2000/regdisparities.doc.

[54] Rodríguez-Oreggia, E, 2005. Regional Disparities and Determinants of Growth in Mexico. Annals of Regional Science 39, 207-220.

[55] Rodríguez-Oreggia, E. (2007). Winners and Losers of Regional Growth in Mexico and their Dynamics. Investigación Económica, 66(259), 43- 62.

[56] Rodríguez-Pose, A. y Sánchez-Reaza, J. (2002a). The impact of trade liberalization on regional disparities in Mexico. Growth and Change, 33 (2002), pp. 72-90

[57] Rodríguez-Pose A. y Sánchez-Reaza J. (2005). Economic polarization through trade: trade liberalization and regional growth in Mexico. In: Kanbur R, Venables JA (eds) Spatial inequality and development. Oxford University Press

[58] Sánchez-Reaza J. y Rodríguez-Pose A. (2002). The impact of trade liberalization on regional disparities in Mexico. Growth Change 33(1):72-90.

[59] Sul, Donggyu (2019). Panel Data Econometrics, Common Factor Analysis for Empirical Researchers. Routledge.

[60] Trejo Nieto, A. (2020). Regional disparities in Mexico and the spatially cumulative effects of national development and economic cycles, 1940-2013. Reg Sci Policy Pract. 2020; 1- 14. https://doi.org/10.1111/rsp3.12311

[61] Valdez, Rolando I. (2019). Spatial diffusion of economic growth and externalities in Mexico. Investigaciones Regionales - Journal of Regional Research, 45: 139-160.

[62] Villarreal C.C. y Tykhonenko A. (2007). Convergencia regional e inversión extranjera directa en México en el contexto del TLCAN, 1994-2002. Investigación Económica:15-41. 